\title{
Box-Particle Labeled Multi-Bernoulli Filter for Multiple Extended Target Tracking
}

\author{
Miao LI, Zaiping LIN, Wei AN, Yiyu ZHOU \\ College of Electronic Science and Engineering, National University of Defense Technology, No. 109, Deya Road, \\ Changsha, P. R. China
}

lm8866@foxmail.com, linzaiping@sina.com, nudtanwei@tom.com, zhouyiyu@sohu.com

Manuscript received January 22, 2016

\begin{abstract}
This paper focuses on real-time tracking of multiple extended targets in clutter based on labeled multiBernoulli filter. To address this problem, a novel approach is proposed within the recently presented box-particle framework. Unlike the traditional point-particle approach, the measurements of extended targets are modeled as interval measurements in this work, and the corresponding likelihood function is given based on interval analysis. Then, labeled multi-Bernoulli recursion for extended targets is implemented by box particles, referred to as BP-LMB filter. Furthermore, BP-MM-LMB filter is proposed to better accommodate the uncertainty of target dynamics by integrating the BP-LMB filter with interacting multiple models (IMM) algorithm. Simulations demonstrate that the proposed approach can significantly reduce the number of particles and well track multiple extended targets with less runtime.
\end{abstract}

\section{Keywords}

Box particle, labeled multi-Bernoulli, multi-target tracking, extended target, interacting multiple models.

\section{Introduction}

Standard tracking algorithms assume that one target generates at most one point measurement. However, in many practical situations this assumption is not appropriate [1], [2]. Due to the increasing resolution of radar and optical sensors, one target may produce multiple point measurements. Such target is referred to as extended target. Extended target tracking is a prevalent task. Recently, the random-finite-set (RFS) based multi-target tracking algorithms have attracted extensive attention [3]. Based on RFS framework, the probability hypothesis density (PHD), Cardinalized PHD (CPHD) and multi-target multi-Bernoulli (MeMBer) filters have been proposed [4-6]. However, these filters only provide unlabeled estimates at each time, and additional post-processing is needed to form target tracks. To overcome the issue, the labeled RFS is introduced in [7] by augmenting the state of each target with a track label. Subsequently, the Generalized Labeled Multi-Bernoulli (GLMB) and the $\delta$-GLMB RFS were proposed as the specific subclasses of labeled RFS [8]. As an accurate and computationally efficient approximation of the $\delta$-GLMB filter, the labeled Multi-Bernoulli (LMB) filter has been proposed in [9]. Both Gaussian Mixtures (GM) and sequential Monte Carlo (SMC) techniques have been implemented for these RFS based filters [6], [10]. The traditional SMC is carried out by point particles (PP). The GM implementation is constrained to linear Gaussian scenarios, and the SMC implementation can be applied to some nonlinear non-Gaussian scenarios. However, in many practical applications, the targets usually exhibit strong maneuver, and the combination of interacting multiple models (IMM) algorithm and RFS based filter is adopted to cope with such rapidly maneuvering targets [11]. In the IMM algorithm, multiple single-model filters are needed. Consequently, the traditional SMC implementation is computationally expensive, especially when the number of targets is large or the IMM algorithm is adopted. This motivates us to search for computationally cheaper alternatives.

For interval measurements, the box-particle (BP) approach has a potential to significantly reduce computational cost [12]. Thus, we focus on tracking multiple extended targets by box-particle approach in this work. In tracking applications, the measurements may be affected by three sources of uncertainty: stochastic, data association and settheoretic uncertainty [13], [14]. The uncertainty originating from random noise is referred to as statistical uncertainty. The data association uncertainty is the uncertainty as to which measurements are corresponding to the target. Traditional tracking algorithms, such as PHD filter and LMB filter, have been designed based on both stochastic and association uncertainty. However, they ignore the effect brought by set-theoretic uncertainty. Because of unknown biases and other reasons, interval measurements, rather than traditional point measurements, can be usually obtained. The box-particle approach based on the interval analysis framework can well accommodate above triple uncertainty. In the Bayesian perspective, box particles can be interpreted as supports of uniform probability density functions (PDF). 
In fact, the interval measurements are common for extended targets. An extended target can generate several point measurements based on Poisson model, and they randomly distribute around the center of target extent [2]. Consequently, the unambiguous position coordinates of target center cannot be extracted. On the contrary, the interval measurements or ambiguous target regions are available.

In this paper, we apply box-particle approach to implement the LMB recursion for extended targets. Firstly, the interval measurement model of extended target is modeled, and the likelihood function within interval analysis framework is given. Secondly, the box-particle implementation of LMB filter for linear extended targets (BP-LMB filter) is derived. Then, to better accommodate maneuvering extended targets, the BP-LMB filter and interacting multiple models (IMM) algorithm are combined, and BP-MM-LMB filter is proposed. Simulations demonstrate that the proposed approach can reach similar accuracy with considerably less computational costs in comparison with point-particle approach.

The remainder of the paper is organized as follows: Section 2 reviews the theories of Poisson model of extended target, LMB filter and interval analysis. Section 3 proposes the BP-LMB filter and BP-MM-LMB filter. The results and analysis of the experiments are mainly presented in Section 4. Section 5 draws conclusions.

\section{Background}

This section provides a brief review of Poisson model of extended target, LMB filter and interval analysis. More details can be found in [2], [9], [12].

\subsection{Poisson Model of Extended Target}

In the multi-target tracking scenario, the tracking is often performed on point measurements after threshold segmentation. The target measurement set of extended target is usually modeled by Poisson model. The cardinality distribution is [2]

$$
\rho(n)=\frac{e^{-\lambda} \lambda^{n}}{n !}
$$

where $\lambda$ is the mean number of point measurements. The point measurements originating from the extended target can be randomly drawn from Gauss spatial distribution around target center.

\subsection{LMB Filter}

A multi-Bernoulli RFS $X$ can be seen as a union of some independent Bernoulli RFSs, i.e. $X=\left\{\left(r^{(i)}, p^{(i)}\right)\right\}_{i=1}^{M}$. Here, $r$ and $p$ represent the existence probability and spatial distribution of single target, respectively. In LMB filter, a label $\ell \in \mathbb{L}$ is appended to single-target state $x \in \mathbb{X}$ to enable the estimation of a target track. Labeled single-target state and multi-target state are denoted by $\mathrm{x}$ and $\mathrm{X}$, respectively. The recursion of LMB filter is carried out on $\mathbb{X} \times \mathbb{L}$ as follows [9].

1) Prediction: Assume that the parameter set of posterior multi-target density at time $k-1$ is given by $\pi_{k-1}=\left\{\left(r_{k-1}^{(\ell)}, p_{k-1}^{(\ell)}\right)\right\}_{\ell \in \mathbb{L}_{k-1}}$. The state space and label space are represented by $\mathbb{X}_{k-1}$ and $\mathbb{L}_{k-1}$. The predicted multitarget LMB RFS parameter set is given as

$$
\pi_{+}=\left\{\left(r_{+, P}^{(\ell)}, p_{+, P}^{(\ell)}\right)\right\}_{\ell \in \mathbb{L}_{k-1}} \cup\left\{\left(r_{B}^{(\ell)}, p_{B}^{(\ell)}\right)\right\}_{\ell \in \mathbb{B}} .
$$

The first term in (2) represents surviving targets and the second one denotes birth targets. $\mathbb{B}$ is the label space of birth targets. The existence probabilities and spatial distributions of surviving targets are calculated as

$$
\begin{gathered}
r_{+, P}^{(\ell)}=\eta_{P}(\ell) r_{k-1}^{(\ell)}, \\
p_{+, P}^{(\ell)}=\left\langle p_{S}(\cdot, \ell) f(x \mid \cdot, \ell), p_{k-1}^{(\ell)}\right\rangle / \eta_{P}(\ell), \\
\eta_{P}(\ell)=\left\langle p_{S}(\cdot, \ell), p_{k-1}^{(\ell)}\right\rangle
\end{gathered}
$$

where the survival probability of the track $\ell$ is represented by $p_{S}(\cdot, \ell)$, and $f(x \mid \cdot, \ell)$ denotes the single-target Markov transition density. The parameter set of birth targets is given by birth model [9].

2) Update: The label space after prediction includes two parts: surviving targets and birth targets, so $\mathbb{L}_{+}=\mathbb{L}_{k-1}+\mathbb{B}$. The predicted LMB parameter set is rewritten as a uniform LMB parameter set as following

$$
\pi_{+}=\left\{\left(r_{+}^{(\ell)}, p_{+}^{(\ell)}\right)\right\}_{\ell \in \mathbb{L}_{+}} .
$$

The LMB filter is an approximation of the $\delta$-GLMB filter, and its measurement update is still carried out in the approach of $\delta$-GLMB. To facilitate the $\delta$-GLMB update, the predicted LMB RFS is converted into an equivalent $\delta$-GLMB form, i.e.

$$
\begin{gathered}
\pi(\mathrm{X})=\Delta(\mathrm{X}) v(\mathcal{L}(\mathrm{X})) p^{\mathrm{X}}, \\
v(L)=\prod_{i \in \mathbb{L}_{+}}\left(1-r^{(i)}\right) \prod_{\ell \in L} \frac{1_{\mathbb{L}_{+}}(\ell) r^{(\ell)}}{1-r^{(\ell)}}, \\
p(x, \ell)=p^{(\ell)}(x)
\end{gathered}
$$

where $\mathcal{L}(\mathrm{x})=\ell$ represents the projection from the labeled state space to the label space, and $\Delta(\mathrm{X})=\delta_{|\mathrm{X}|}(|\mathcal{L}(\mathrm{X})|)$.

Before update, measurement set partitioning is needed for extended target tracking, which partitions point measurement set into multiple subsets. A well-known partition- 
ing method is the distance partition. It is carried out based on the fundamental insight that measurements from the same extended target are spatially close to each other [15]. In particle applications, the distance threshold can be set based on prior knowledge, and then the most likely ones among all of the possible partitions are adopted in measurement update.

Afterwards, the updated existence probabilities and spatial distributions can be computed by:

$$
\begin{gathered}
r^{(\ell)}=\sum_{\left(I_{+}, \theta\right) \in \mathcal{F}\left(\mathbb{L}_{+}\right) \times \Theta_{I_{+}}} v^{\left(I_{+}, \theta\right)}(Z) 1_{I_{+}}(\ell), \\
p^{(\ell)}=\frac{1}{r^{(\ell)}} \sum_{\left(I_{+}, \theta\right) \in \mathcal{F}\left(\mathbb{L}_{+}\right) \times \Theta_{I_{+}}} v^{\left(I_{+}, \theta\right)}(Z) 1_{I_{+}}(\ell) p^{(\theta, \ell)}(x)
\end{gathered}
$$

where $\Theta_{I_{+}}$represents the space of mappings from track label to measurement, and $\theta: I_{+} \rightarrow\{0,1, \cdots,|Z|\} \cdot 1_{Y}(X)$ is include indicator function [9].

\subsection{Interval Analysis}

Measurements with bounded errors can be conveniently modeled as interval measurements. To facilitate the processing of interval measurements, the tool of interval analysis has been developed [12]. A real interval is defined as a closed and connected subset, i.e. $[x]=[\underline{x}, \bar{x}]$. The $\underline{x}$ and $\bar{x}$ represent respectively the lower bound and upper bound. For the case with multi-dimensional states, the interval is regarded as a box $[\mathrm{x}]$. Box $[\mathrm{x}]$ is defined as a Cartesian product of multiple intervals, i.e. $[\mathrm{x}]=\left[x_{1}\right] \times\left[x_{2}\right] \cdots \times\left[x_{d}\right]$, where $d$ is the number of dimensions. After a nonlinear propagation, the result of the propagation may not be a box. To cope with this issue, inclusion functions are necessary. Let $f$ be a function from $\mathbb{R}^{n}$ to $\mathbb{R}^{m}$. An interval function $[f]$ from $\mathbb{\mathbb { R } ^ { n }}$ to $\mathbb{I} \mathbb{R}^{m}$ is said to be an inclusion function for $f$, if $f([\mathrm{x}]) \subseteq[f]([\mathrm{x}])$, $\forall[\mathrm{x}] \in \mathbb{I R}^{n}$. In the context of tracking, natural inclusion function is usually adopted [12], [16].

The contraction is another important concept for boxparticle approach, which is used in the calculation of likelihood function as given in Sec. 3.1. A Constraint Satisfaction Problem (CSP) can be denoted by

$$
\mathcal{H}=(g(\mathrm{x})=0, \mathrm{x} \in[\mathrm{x}]) .
$$

In short, equation (12) means finding the smallest box $[\mathrm{x}]^{\prime}$ to replace original $[\mathrm{x}]$ under the constraint $\mathbb{S} \in[\mathrm{x}]^{\prime} \in[\mathrm{x}]$. The Constraint Propagation (CP) has better suitability in comparison with other methods. Thus, the $\mathrm{CP}$ method has been widely used in many tracking applications [14], [16].

\section{Box-Particle Implementations}

\subsection{Interval Measurement Model and Likelihood Function}

For a two-dimensional case, the single-target state can be described by interval state vector as

$$
[\mathrm{x}]=\left(\left[x_{k}\right],\left[\dot{x}_{k}\right],\left[y_{k}\right],\left[\dot{y}_{k}\right]\right)^{T}
$$

where $\left[x_{k}\right],\left[y_{k}\right]$ are the position intervals of the target center, and $\left[\dot{x}_{k}\right]$ and $\left[\dot{y}_{k}\right]$ are the corresponding velocity intervals.

For an extended target, some point measurements around target center can be obtained after threshold segmentation, i.e. $Z=\left\{\left(x^{i}, y^{i}\right)\right\}_{i=1}^{M}$. Here, the term $M$ represents the number of point measurements originating from the extended target, and $x^{i}$ and $y^{i}$ are the position coordinates of the $i$ th point measurement. As introduced in Sec. 2.1, the $M$ is subject to Poisson distribution. Obviously, the precise center of target extent cannot be directly obtained. However, the interval at which the target center locates is available, i.e. $[\mathrm{z}]=\left(\left[\mathrm{z}_{x}\right],\left[\mathrm{z}_{y}\right]\right)$. The $\left[\mathrm{z}_{x}\right]$ and $\left[\mathrm{z}_{y}\right]$ are interval measurements of target center in $\mathrm{X}$-direction and $y$-direction, respectively. In this work, they are computed by

$$
\begin{aligned}
& {\left[\mathrm{z}_{x}\right]=x_{C}+\left[-\varepsilon_{x},+\varepsilon_{x}\right],} \\
& {\left[\mathrm{z}_{y}\right]=y_{C}+\left[-\varepsilon_{y},+\varepsilon_{y}\right]}
\end{aligned}
$$

where $x_{C}$ and $y_{C}$ are the estimated position coordinates of target center, $\varepsilon_{x}$ and $\varepsilon_{y}$ describe the size of interval. They are computed by:

$$
\begin{gathered}
x_{C}=\operatorname{mean}\left(\left\{x^{i}\right\}_{i=1}^{M}\right), \\
y_{C}=\operatorname{mean}\left(\left\{y^{i}\right\}_{i=1}^{M}\right), \\
\varepsilon_{x}=\frac{\max \left(\left\{x^{i}\right\}_{i=1}^{M}\right)-x_{C}}{2}, \\
\varepsilon_{y}=\frac{\max \left(\left\{y^{i}\right\}_{i=1}^{M}\right)-y_{C}}{2} .
\end{gathered}
$$

For convenience, the above processing, from point measurement set to one interval measurement, is denoted by $[\mathrm{z}]=\mathcal{F}_{I}(Z)$.

The measurement likelihood function $g(z \mid x)$ is required by Bayes-like filters. This is also true for box-parti- 
cle filter. In the context of this paper, the measurement likelihood for interval measurement $[z]$ is given as

$$
g\left([\mathrm{z}][[\mathrm{x}])=\frac{\left|\left[\mathrm{h}_{\mathrm{CP}}\right]([\mathrm{x}],[\mathrm{z}])\right|}{|[\mathrm{x}]|}\right.
$$

where the function $\left[\mathrm{h}_{\mathrm{CP}}\right]([\mathrm{x}],[\mathrm{z}])$ returns a contracted version of $[\mathrm{x}]$. In this work, the directly measured components are contracted based on interval measurements, i.e. $[x]=[x] \cap\left[z_{x}\right],[y]=[y] \cap\left[z_{y}\right]$. The unmeasured components, $[\dot{x}]$ and $[\dot{y}]$, are contracted based on prior knowledge. $|[\mathrm{x}]|=|[x]| \times|[\dot{x}]| \times[[y]|\times|[\dot{y}] \mid$ where $|\cdot|$ denotes the volume of interval.

\subsection{BP-LMB Filter}

In this subsection, the box-particle implement of LMB filter is derived in detail, which is referred to as BP-LMB filter in this paper.

In classical LMB filter, the spatial distribution $p(\mathrm{x})$ is approximated using a set of weighted point particles as

$$
p(\mathrm{x}) \approx \sum_{j=1}^{J} \omega^{(j)} \delta_{\mathrm{x}^{(j)}}(\mathrm{x})
$$

where $\delta_{\mathrm{x}^{(j)}}(\mathrm{x})$ denotes the Dirac delta function concentrated at $\mathrm{x}^{(j)}, J$ is the number of point particles, and $\omega^{(j)}$ represents the weight of the $j$ th point particle.

In box particle approach, box particles are interpreted as supports of uniform PDF, then equation (21) is rewritten as:

$$
p(\mathrm{x}) \approx \sum_{j=1}^{J} \omega^{(j)} U_{\left[\mathrm{x}^{(j)}\right]}(\mathrm{x})
$$

where $U_{\left[\mathrm{x}^{(j)}\right]}(\mathrm{x})$ represents the uniform PDF over the box $\left[\mathrm{x}^{(j)}\right]$. The details about BP-LMB recursion are presented as following.

1) Prediction: Suppose that the posterior multi-target density at $k-1$ is an LMB RFS with parameter set $\pi_{k-1}=\left\{\left(r_{k-1}^{(\ell)}, p_{k-1}^{(\ell)}\right)\right\}_{\ell \in \mathbb{L}_{k-1}}, p_{k-1}^{(\ell)}$ is approximated by a set of weighted box particles $\left\{\omega_{k-1}^{(\ell, j)},\left[\mathrm{x}_{k-1}^{(\ell, j)}\right]\right\}_{j=1}^{J_{k-1}^{(\ell)}}$, i.e.

$$
p_{k-1}^{(\ell)}(\mathrm{x}) \approx \sum_{j=1}^{J_{k-1}^{(\ell)}} \omega_{k-1}^{(\ell, j)} U_{\left[\mathrm{x}_{k-1}^{(\ell, j)}\right]}(\mathrm{x})
$$

where $J_{k-1}^{(\ell)}$ is the number of box particles of track $\ell$ at time $k-1$.

The predicted LMB density consists of surviving extended targets and birth extended targets in (2). In the
BP-LMB filter, the parameter set of surviving extended targets can be calculated by

$$
\begin{gathered}
r_{+, P}^{(\ell)}=r_{k-1}^{(\ell)} \sum_{j=1}^{J_{k-1}^{(\ell)}} \omega_{k-1}^{(\ell, j)} p_{S}, \\
p_{+, P}^{(\ell)}(\mathrm{x})=\sum_{j=1}^{J_{k-1}^{(\ell)}} \tilde{\omega}_{+, P}^{(\ell, j)} U_{\left[\mathrm{x}_{+, P}^{(\ell, j)}\right]}(\mathrm{x}),
\end{gathered}
$$

where

$$
\begin{gathered}
{\left[\mathrm{x}_{+, P}^{(\ell, j)}\right]=[f]\left(\left[\mathrm{x}_{k-1}^{(\ell, j)}\right]\right),} \\
\tilde{\omega}_{+, P}^{(\ell, j)}=\omega_{k-1}^{(\ell, j)} p_{S} / \sum_{j=1}^{J_{k-1}^{(\ell)}}\left\{\omega_{k-1}^{(\ell, j)} p_{S}\right\} .
\end{gathered}
$$

The survival probability $p_{S}$ is assumed to be state independent. The output of $[f]([\mathrm{x}])$ is a box containing $f([\mathrm{x}])$. The parameter set of birth extended targets are given by birth model in Sec. 4.1.

2) Update: After prediction, the uniform multi-target density $\pi_{+}=\left\{\left(r_{+}^{(\ell)}, p_{+}^{(\ell)}\right)\right\}_{\ell \in \mathbb{L}_{+}}$is given, and each $p_{+}^{(\ell)}$ is approximated by a set of box particles $\left\{\omega_{+}^{(\ell, j)},\left[\mathrm{x}_{+}^{(\ell, j)}\right]\right\}_{j=1}^{J_{+}^{(\ell)}}$, i.e.

$$
p_{+}^{(\ell)}(\mathrm{x}) \approx \sum_{j=1}^{J_{(+)}^{(\ell)}} \omega_{+}^{(\ell, j)} U_{\left[\mathrm{x}_{+}^{(\ell, j)}\right]}(\mathrm{x}) .
$$

Then, the equivalent $\delta$-GLMB form is obtained by ( 7 to 9). Based on distance partition as introduced in Sec. 2.2, the point measurement set is divided into disjointed subsets, i.e. $Z_{k}=\left\{Z_{k, s u b}^{(n)}\right\}_{n=1}^{N} . Z_{k, s u b}^{(n)}$ represents the $n$th subset and it consists of some point measurements, i.e. $Z_{k, s u b}^{(n)}=$ $\left\{\left(x_{k}^{(n, m)}, y_{k}^{(n, m)}\right)\right\}_{m=1}^{M^{(n)}}$. The distance threshold $T h_{p a r}$ used in distance partition is specified a priori. Then, subsets are converted into interval measurements using the method introduced in Sec. 3.1, i.e. $\left[\mathrm{Z}_{k}\right]=\left\{\left[\mathrm{z}_{k}^{(n)}\right]\right\}_{n=1}^{N}$ where $\left[\mathrm{z}_{k}^{(n)}\right]=\mathcal{F}_{I}\left(Z_{k, s u b}^{(n)}\right)$.

The update using the interval measurement set $\left[Z_{k}\right]$ is given by

$$
\begin{gathered}
r^{(\ell)}=\sum_{\left(I_{+}, \theta\right) \in \mathcal{F}\left(\mathbb{L}_{+}\right) \times \Theta_{I_{+}}} v^{\left(I_{+}, \theta\right)}\left(\left[\mathrm{Z}_{k}\right]\right) 1_{I_{+}}(\ell), \\
p^{(\ell)}=\frac{1}{r^{(\ell)}} \sum_{\left(I_{+}, \theta\right) \in \mathcal{F}\left(\mathbb{L}_{+}\right) \times \Theta_{I_{+}}} v^{\left(I_{+}, \theta\right)}\left(\left[\mathrm{Z}_{k}\right]\right) 1_{I_{+}}(\ell) p^{(\theta, \ell)}(\mathrm{x}) .
\end{gathered}
$$

The weights and the spatial distributions are calculated by

$$
v^{\left(I_{+}, \theta\right)}\left(\left[\mathrm{Z}_{k}\right]\right) \propto v_{+}^{\left(I_{+}\right)}\left[\eta_{\left[Z_{k}\right]}^{(\theta)}\right]^{I_{+}},
$$




$$
\begin{aligned}
& p^{(\theta, \ell)}(\mathrm{x})=\sum_{j=1}^{J_{t}^{(\ell)}} \tilde{\omega}_{j}^{(\theta, \ell)} U_{\left[\mathrm{x}_{j}^{(\theta, \ell)}\right]}(\mathrm{x}), \\
& \mathrm{x}_{j}^{(\theta, \ell)}=\left[\mathrm{h}_{\mathrm{CP}}\right]\left(\left[\mathrm{x}_{+}^{(\ell, j)}\right],\left[\mathrm{z}_{k}^{\theta(\ell)}\right]\right), \\
& \tilde{\omega}_{j}^{(\theta, \ell)}=\frac{\omega_{+}^{(\ell, j)} \psi_{\left[\mathrm{Z}_{k}\right]}\left(\left[\mathrm{x}_{+}^{(\ell, j)}\right] ; \theta\right)}{\eta_{\left[\mathrm{Z}_{k}\right]}^{(\theta)}(\ell)}, \\
& \eta_{\left[Z_{k}\right]}^{(\theta)}(\ell)=\sum_{j=1}^{J_{+}^{(\ell)}} \omega_{+}^{(\ell, j)} \psi_{\left[Z_{k}\right]}\left(\left[\mathrm{x}_{+}^{(\ell, j)}\right] ; \theta\right), \\
& \psi_{\left[\mathrm{Z}_{k}\right]}\left(\left[\mathrm{x}_{+}^{(\ell, j)}\right] ; \theta\right)= \begin{cases}\frac{p_{D} g\left(\left[\mathrm{z}_{k}^{\theta(\ell)}\right] \mid\left[\mathrm{x}_{+}^{(\ell, j)}\right]\right)}{\kappa}, & \text { if } \theta(\ell)>0 \\
q_{D}, & \text { if } \theta(\ell)=0\end{cases}
\end{aligned}
$$

where $g([\mathrm{z}] \mid[\mathrm{x}])$ is the interval measurement likelihood and has been given as (20). $\kappa$ denotes the clutter density, and the clutter is modeled as Poisson model [9]. $p_{D}$ represents the detection probability, it is state independent, and $q_{D}=1-p_{D}$.

3) Resampling: Similar to the traditional point-particle approach, the resampling step is necessary for the boxparticle approach to prevent degeneracy of box particles. However, instead of replicating box particles with large posterior weights, we partition them into multiple sub boxes. These sub boxes are equally weighted. Consequently, the 'resolution' in the regions of state space can be refined, and target state intervals can be contracted. In this work, we randomly pick a dimension to be divided for the selected box-particle [12].

In addition, the pruning, merging and state extraction are similar to those in [14]. Pruning of hypothesized tracks means that the tracks with existence probabilities below the threshold $T h_{d e l}$ will be deleted. Two tracks within a distance threshold $T h_{m e r}$ will be merged. A target is declared present only if the existence probability is greater than threshold $T h_{T a r}$. Finally, the multi-target state can be extracted by

$$
\mathbf{x}^{(\ell)}=\sum_{j=1}^{J^{(\ell)}} \omega^{(\ell, j)} \operatorname{mid}\left(\left[\mathbf{x}^{(\ell, j)}\right]\right)
$$

where $\operatorname{mid}([\cdot])$ represents finding the center of the box particle $[\mathrm{x}]$.

\subsection{BP-MM-LMB Filter}

To better accommodate maneuvering extended targets, BP-MM-LMB filter is proposed by integrating the BPLMB filter with IMM algorithm.

To derive the BP-MM-LMB filter, $\tau \in \mathbb{T}$ is introduced to denote single motion model. The state of single extended target is consequently represented by $\left(\mathrm{x}^{(\ell)}, \tau\right)$.
The motion model of the target is usually regarded as a Markov chain with transition probability matrix $H=\left[h_{s t}\right]$ [17]. For example, $h_{s t}=p_{k \mid k-1}\left\{\tau_{k}=t \mid \tau_{k-1}=s\right\}$ denotes the probability that a target switches from model $s$ to model $t$, where $s, t \in \mathbb{T}$ and $\sum_{t=1}^{M^{\tau}} h_{s t}=1$. The term $M^{\tau}$ is the number of motion models.

In essence, the BP-MM-LMB filter consists of a finite number of single-model BP-LMB filters corresponding to different motion models. In addition to those steps in BPLMB filter, mixing step is additionally required [18]. The other steps are similar to BP-LMB filter, and the mixing step of BP-MM-LMB filter is given as follows.

Assume that at time $k-1$ the multi-target posterior density is $\pi_{k-1}=\left\{\left(r_{k-1}^{(\ell)}, p_{k-1}^{(\ell)}\left(\left[\mathrm{x}_{k-1}^{(\ell)}\right], \tau_{k-1}^{(\ell)}\right)\right)\right\}_{\ell \in \mathbb{L}_{k-1}}$, and each $p_{k-1}^{(\ell)}$ is approximated by a set of augmented box particles $\left\{\omega_{k-1}^{(\ell, j)},\left[\mathrm{x}_{k-1}^{(\ell, j)}\right], \tau_{k-1}^{(\ell, j)}\right\}_{j=1}^{J_{k-1}^{(\ell)}}$ i.e.
$p_{k-1}^{(\ell)}(\mathrm{x}, \tau) \approx \sum_{j=1}^{J_{k-1}^{(\ell)}} \omega_{k-1}^{(\ell, j)} U_{\left[\mathrm{x}_{k-1}^{(\ell, j)}\right]}(\mathrm{x}) \delta_{\tau_{k-1}^{(\ell, j)}}(\tau)$.

Then, the mixed LMB parameter set can be expressed by:

$$
\tilde{\pi}_{k-1}=\left\{\left(r_{k-1}^{(\ell)}, \tilde{p}_{k-1}^{(\ell)}\left(\left[\mathrm{x}_{k-1}^{(\ell)}\right], \tau_{k}^{(\ell)}\right)\right)\right\}_{\ell \in \mathbb{L}_{k-1}} .
$$

Since the model transition is only decided by model transition probability and is independent of the state transition, $\tilde{p}_{k-1}^{(\ell)}\left(\left[\mathrm{x}_{k-1}^{(\ell)}\right], \tau_{k}^{(\ell)}=t\right)$ can be computed by

$$
\begin{aligned}
& \tilde{p}_{k-1}^{(\ell)}\left(\left[\mathrm{x}_{k-1}^{(\ell)}\right], \tau_{k}^{(\ell)}=t\right) \\
& =\sum_{s=1}^{M^{\tau}} p_{k-1}^{(\ell)}\left(\left[\mathrm{x}_{k-1}^{(\ell)}\right], \tau_{k}^{(\ell)}=t, \tau_{k-1}^{(\ell)}=s\right) \\
& =\sum_{s=1}^{M^{\tau}} p_{k-1}^{(\ell)}\left(\tau_{k}^{(\ell)}=t \mid\left[\mathrm{x}_{k-1}^{(\ell)}\right], \tau_{k-1}^{(\ell)}=s\right) \cdot p_{k-1}^{(\ell)}\left(\left[\mathrm{x}_{k-1}^{(\ell)}\right], \tau_{k-1}^{(\ell)}=s\right) \\
& =\sum_{s=1}^{M^{\tau}} h_{s t} p_{k-1}^{(\ell)}\left(\left[\mathrm{x}_{k-1}^{(\ell)}\right], \tau_{k-1}^{(\ell)}=s\right) .
\end{aligned}
$$

Therefore, $\tilde{p}_{k-1}^{(\ell)}\left(\left[\mathbf{x}_{k-1}^{(\ell)}\right], \tau_{k}^{(\ell)}\right)$ is approximated by

$$
\bigcup_{t=1}^{M^{\tau}}\left\{\omega_{k-1}^{(\ell, j)} h_{\tau_{k-1}^{(\ell, j)}, t},\left[\mathbf{x}_{k-1}^{(\ell, j)}\right], \tau_{k}^{(\ell, j)}=t\right\}_{j=1}^{J_{k-1}^{(\ell)}} .
$$

It can be found that much more particles are needed and the number of particles after mixing for track $\ell$ is increased to $M^{\tau} \times J_{k-1}^{(\ell)}$. Thus, computationally cheaper implementation is important for maneuvering target tracking.

Subsequently, the prediction and update are carried out based on augmented box particles. Note that the augmented single-target state transition density is $f\left([\mathrm{x}], \tau \mid\left[\mathrm{x}^{\prime}\right], \tau^{\prime}\right)=h_{\tau^{\prime} \tau} f\left([\mathrm{x}] \mid\left[\mathrm{x}^{\prime}\right]\right)$. 


\section{Numerical Studies}

\subsection{Simulation Setup}

The simulation results are divided into two parts: Firstly, multiple linear extended targets are tracked by the proposed BP-LMB filter and the traditional point-particle LMB filter (PP-LMB filter) [1], [9]. Secondly, the BPMM-LMB filter is compared with the single-model BPLMB filter (BP-CV-LMB filter) and multi-model PP-LMB filter (PP-MM-LMB filter) in the scenario containing multiple rapidly maneuvering extended targets.

Consider a 2D surveillance region in the Cartesian coordinates, specified by the lower-left corner $(1,1)$ and upper-right corner $(512,512)$. Each sequence has 30 frames and period is $\Delta_{T}=1 \mathrm{~s}$. There are three extended targets in each sequence, and they are embedded into each sequence as Poisson model. The average number of target point measurements is 8 . The average number of clutter measurements is 20 in each frame. In the first experiment, three targets all move at a constant velocity (CV) model in the surveillance region. However, the targets may move at a constant velocity model or a coordinated turn (CT) model in the second experiment.

Similar to classical LMB filter, the birth process is modeled as a multi Bernoulli RFS [9], i.e. $\pi_{B}=\left\{\left(r_{B}^{(\ell)}, p_{B}^{(\ell)}\right)\right\}_{\ell=1}^{3}, r_{B}^{(\ell)}=0.06, p_{B}^{(\ell)}(\mathrm{x}) \approx \omega^{(\ell)} U_{\left[\mathrm{x}_{B}^{(\ell)}\right]}(\mathrm{x})$.

Taking the Target 1 as an example, $\omega^{(1)}=1$ and the initial target state vector is

$$
\mathbf{x}_{B}^{(1)}=([385,399],[-15,15],[155,169],[-15,15]) .
$$

The velocity vector is contracted based on prior distribution. In this work, it follows a uniform PDF, i.e. $[\dot{x}] \in[-15,-5] \cup[5,15]$ and $[\dot{y}] \in[-15,-5] \cup[5,15]$.

The state transition functions of $\mathrm{CV}$ model and CT model are described by (43) and (44) [18]. The turn rate of CT model in the second experiment is set to $\Omega=0.8 \mathrm{rad}$.

$$
\begin{gathered}
f_{C V}=\left[\begin{array}{cccc}
1 & \Delta_{T} & 0 & 0 \\
0 & 1 & 0 & 0 \\
0 & 0 & 1 & \Delta_{T} \\
0 & 0 & 0 & 1
\end{array}\right] \\
f_{C T}=\left[\begin{array}{cccc}
1 & \frac{\sin \Omega \Delta_{T}}{\Omega} & 0 & -\frac{1-\cos \Omega_{T}}{\Omega} \\
0 & \cos \Omega \Delta_{T} & 0 & -\sin \Omega \Delta_{T} \\
0 & \frac{1-\cos \Omega \Delta_{T}}{\Omega} & 1 & \frac{\sin \Omega \Delta_{T}}{\Omega} \\
0 & \sin \Omega \Delta_{T} & 0 & \cos \Omega \Delta_{T}
\end{array}\right]
\end{gathered}
$$

The statistical performance of the algorithm is evaluated using the Optimal Sub-Pattern Assignment (OSPA) metric [19], because it jointly captures differences in cardinality and individual elements between two finite sets in a mathematically consistent yet intuitively meaningful way. Then, for $1 \leq p \leq \infty, \quad c>0, \quad X=\left\{x_{1}, \cdots, x_{m}\right\}$ and $Y=\left\{y_{1}, \cdots, y_{n}\right\}$, if $m \leq n$,

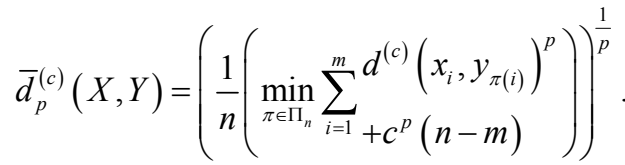

If $\quad m>n, \quad \bar{d}_{p}^{(c)}(X, Y)=\bar{d}_{p}^{(c)}(Y, X) \quad$. If $\quad m=n=0$, $\bar{d}_{p}^{(c)}(X, Y)=0$. We use the parameters $c=50$ and $p=1$ in this work. Some parameters used in LMB recursion are listed in Tab. 1.

\begin{tabular}{|c|c|c|c|c|c|c|}
\hline Variable & $p_{S}$ & $p_{D}$ & $T h_{m e r}$ & $T h_{\text {del }}$ & $T h_{T a r}$ & $T h_{p a r}$ \\
\hline Value & 0.99 & 0.98 & 3 & $10^{-10}$ & 0.5 & 5 \\
\hline
\end{tabular}

Tab. 1. Parameters of LMB recursion.

\subsection{Tracking Multiple Linear Extended Targets}

In this experiment, there are three extended targets in this scenario, and they all move at a CV model. The typical point measurements originating from the Target 1 are given in Fig. 1. The true target tracks are shown in Fig. 2. Target 1, Target 2 and Target 3 are born at $1 \mathrm{~s}, 10 \mathrm{~s}$ and $20 \mathrm{~s}$, respectively.

The tracking results obtained from PP-LMB filter and the proposed BP-LMB filter for a single run is also given in Fig. 2. It can be seen that two filters can successfully track three linear extended targets. To provide a performance comparison in sense of statistical evaluation, the average cardinality and average OPSA distance over 50 Monte Carlo runs are shown in Fig. 3 and Fig. 4, respectively.

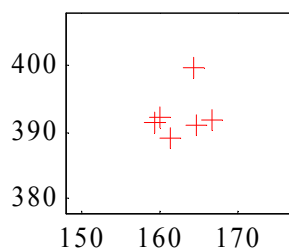

(a) Frame 1

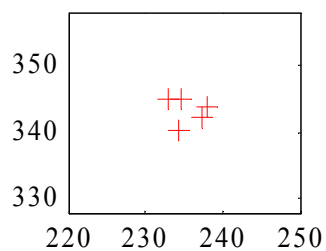

(b) Frame 7

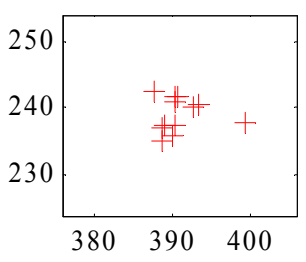

(c) Frame 20

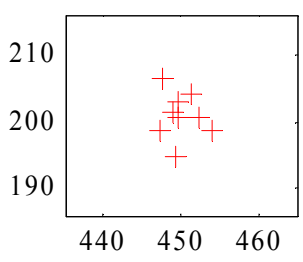

(d) Frame25
Fig. 1. The point measurements of Target 1 at different frames in the first experiment. 


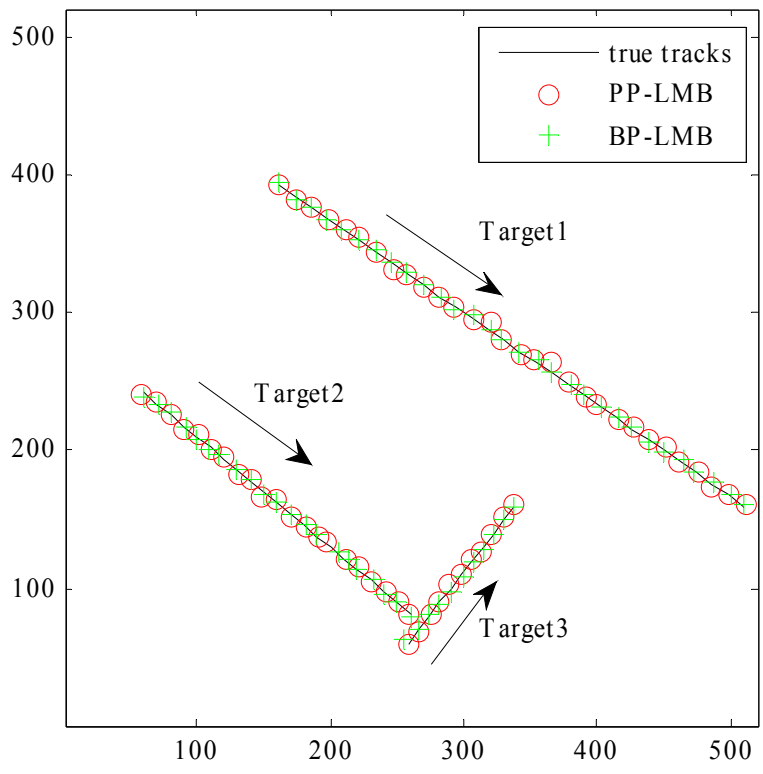

Fig. 2. The true tracks and tracking results in the first experiment.

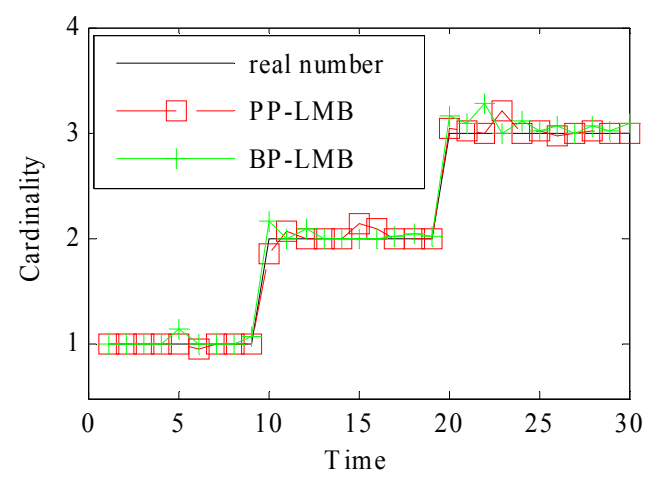

Fig. 3. Cardinality statistics for PP-LMB and BP-LMB filters in the first experiment.

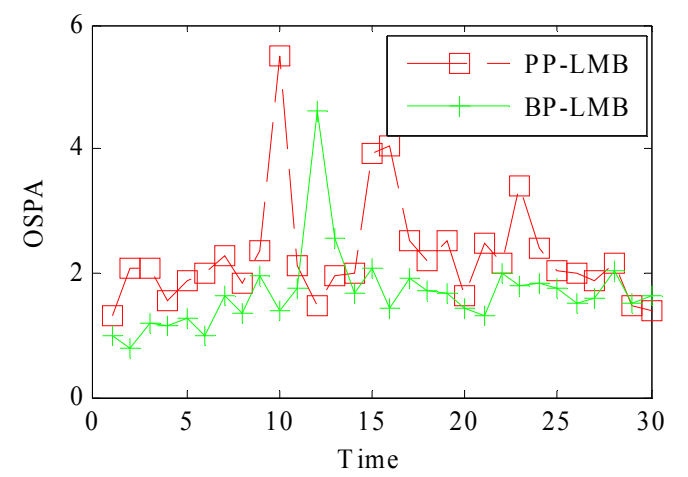

Fig. 4. Average OSPA distances for PP-LMB and BP-LMB filters in the first experiment.

\begin{tabular}{|c|c|c|}
\hline Parameter & PP-LMB & BP-LMB \\
\hline $\begin{array}{c}\text { Surviving } \\
\text { particle number }\end{array}$ & 2000 & 40 \\
\hline $\begin{array}{c}\text { Newborn } \\
\text { particle number }\end{array}$ & 1000 & 1 \\
\hline Runtime (sec) & 786.27 & 43.03 \\
\hline
\end{tabular}

Tab. 2. Average runtime and the number of particles for PP-LMB and BP-LMB filters in the first experiment.
For RFS based algorithms, the cardinality means the number of estimated targets. The OSPA distance is a proper metric, which penalizes both the cardinality error and the error in the state space. As shown in Fig. 3, both PP-LMB filter and BP-LMB filter can accurately estimate cardinality. However, the proposed BP-LMB filter can achieve more accurate state estimation. The OSPA peak appears at about $10 \mathrm{~s}$, because of birth targets. As shown in Tab. 2, 40 box particles are enough for each track in the proposed BP-LMB filter. However, PP-LMB filter requires 2000 point particles. The number of particles needed for BP-LMB filter is much smaller than that of PP-LMB filter. As a result, the average runtime of BP-LMB filter is much less than that of PP-LMB filter. It proves the proposed BPLMB filter can remarkably decreases the runtime for multiple linear extended targets.

\subsection{Tracking Multiple Maneuvering Extended Targets}

In this experiment, true target tracks are shown in Fig. 5. There are two highly maneuvering extended targets and one linear extended target in this scenario. For example, Target 1 is born at $1 \mathrm{~s}$ and dies at $30 \mathrm{~s}$. It firstly moves at $\mathrm{CV}$ model from $1 \mathrm{~s}$ to $9 \mathrm{~s}$, then executes CT model from the $10 \mathrm{~s}$ to $12 \mathrm{~s}$, and finally moves at CV model from $13 \mathrm{~s}$ to end. The motions of the targets are summarized in Tab. 3.

Let $\tau=1$ denote the $\mathrm{CV}$ model and $\tau=2$ denote the CT model. The initial motion models are set to CV. The model transition matrix is set to

$$
H=\left[\begin{array}{ll}
0.85 & 0.15 \\
0.15 & 0.85
\end{array}\right]
$$

The tracking results obtained from PP-MM-LMB, BP-CV-LMB and BP-MM-LMB filters for a single run are also shown in Fig. 5. When the motion model switch occurs, the single-model filter fails to capture the targets. However, the PP-MM-LMB filter and BP-MM-LMB filter can well accommodate target maneuver.

The average cardinality and the average OPSA distance over 50 Monte Carlo runs are depicted in Fig. 6 and 7. BP-CV-LMB filter yields large cardinality error at $10 \mathrm{~s}$ to $20 \mathrm{~s}$ and $26 \mathrm{~s}-30 \mathrm{~s}$ due to target maneuver. Furthermore, there are high peaks in OSPA metric where the estimated cardinality is incorrect. It proves that single-model filter is not enough to track rapidly maneuvering extended targets.

\begin{tabular}{|c|c|c|c|c|}
\hline Target & $\begin{array}{c}\text { Born } \\
\text { time }\end{array}$ & $\begin{array}{c}\text { Die } \\
\text { time }\end{array}$ & $\begin{array}{c}\text { CV } \\
\text { motion }\end{array}$ & $\begin{array}{c}\text { CT } \\
\text { motion }\end{array}$ \\
\hline Target 1 & $1 \mathrm{~s}$ & $30 \mathrm{~s}$ & $\begin{array}{c}1 \mathrm{~s}-9 \mathrm{~s}, \\
13 \mathrm{~s}-30 \mathrm{~s}\end{array}$ & $10 \mathrm{~s}-12 \mathrm{~s}$ \\
\hline Target 2 & $10 \mathrm{~s}$ & $30 \mathrm{~s}$ & $\begin{array}{c}1 \mathrm{~s}-25 \mathrm{~s}, \\
29 \mathrm{~s}-30 \mathrm{~s}\end{array}$ & $26 \mathrm{~s}-28 \mathrm{~s}$ \\
\hline Target 3 & $20 \mathrm{~s}$ & $30 \mathrm{~s}$ & $1 \mathrm{~s}-30 \mathrm{~s}$ & \\
\hline
\end{tabular}

Tab. 3. The motion of extended targets in the second experiment. 


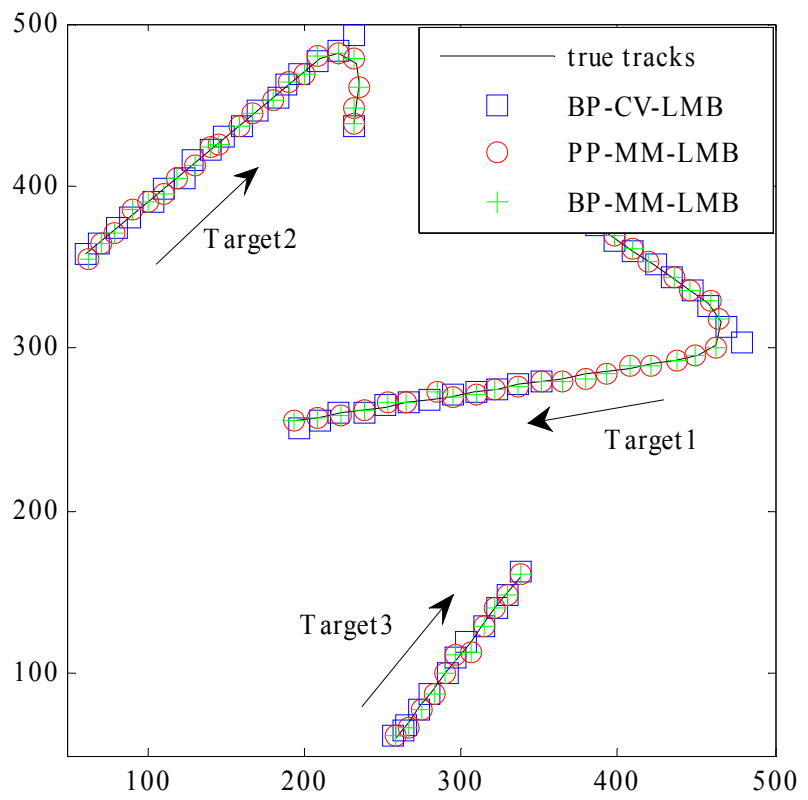

Fig. 5. The true tracks and tracking results in the second experiment.

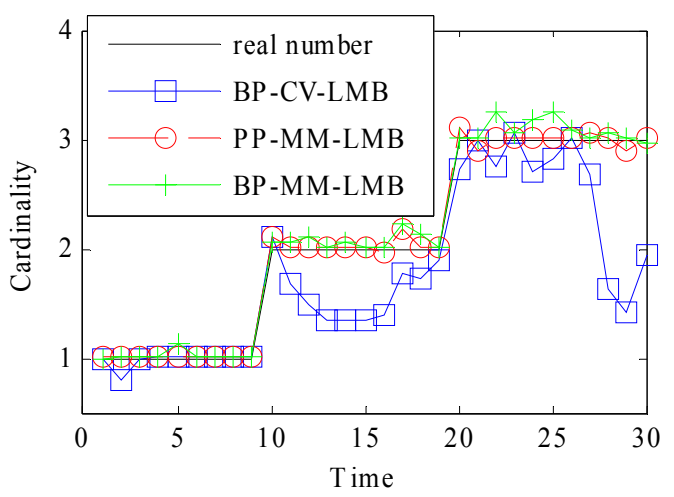

Fig. 6. Cardinality statistics for BP-CV-LMB, PP-MM-LMB and BP-MM-LMB filters in the second experiment.

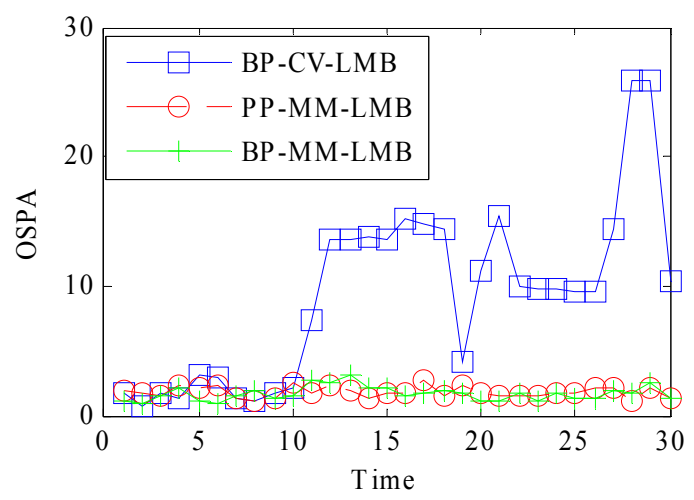

Fig. 7. Average OSPA distances for BP-CV-LMB, PP-MMLMB and BP-MM-LMB filters in the second experiment.

As indicated by Fig. 7, both PP-MM-LMB filter and BP-MM-LMB filter perform comparably well. However, much fewer particles are needed by the BP-MM-LMB filter. The average runtimes of these three filters are listed in Tab. 4. The average runtime of BP-MM-LMB filter is

\begin{tabular}{|c|c|c|c|}
\hline $\begin{array}{c}\text { Filter } \\
\text { Parameter }\end{array}$ & $\begin{array}{c}\text { PP- } \\
\text { MM-LMB }\end{array}$ & $\begin{array}{c}\text { BP- } \\
\text { CV-LMB }\end{array}$ & $\begin{array}{c}\text { BP- } \\
\text { MM-LMB }\end{array}$ \\
\hline $\begin{array}{c}\text { Surviving } \\
\text { particle number }\end{array}$ & 2000 & 40 & 40 \\
\hline $\begin{array}{c}\text { Newborn } \\
\text { particle umber }\end{array}$ & 1000 & 1 & 1 \\
\hline Runtime(sec) & 909.35 & 41.18 & 75.59 \\
\hline
\end{tabular}

Tab. 4. Average runtime and the number of particles in the second experiment.

$75.59 \mathrm{~s}$, and it is about ten times less than that of PP-MMLMB filter. It proves that BP-MM-LMB filter is more computationally efficient than PP-MM-LMB filter. Therefore, the proposed approach is more appropriate for realtime tracking.

\section{Conclusions}

This paper presents a novel approach to track multiple extended targets within the box-particle framework. BPLMB and BP-MM-LMB filters are proposed to track linear extended targets and rapidly maneuvering extended targets, respectively. In our approach, the measurements of extended target are modeled as interval measurements, and the point samples are replaced by region samples in LMB recursion. Compared with traditional point-particle approach, the proposed approach needs fewer particles to guarantee a similar accuracy. Consequently, multiple extended targets can be tracked using less runtime, which is important for real-time applications.

\section{References}

[1] CAI, F., FAN, H., FU, Q. Bernoulli filter for extended target in clutter using Poisson models. Chinese Journal of Electronics, 2015, vol. 24, no. 2, p. 326-331. DOI: 10.1049/cje.2015.04.017

[2] GILHOLM, K., GODSILl, S., MASKELl, S., et al. Poisson models for extended target and group tracking. In SPIE Proc. 5913. California (USA), 2005, p. 59130R-59130R12. DOI: $10.1117 / 12.618730$

[3] MAHLER, R. P. S. Statistical Multisource Multitarget Information Fusion. London (UK): Artech House, 2007.

[4] MAHLER, R. P. S. Multitarget Bayes filtering via first-order multitarget moments. IEEE Transactions on Aerospace and Electronic Systems, 2003, vol. 39, no. 4, p. 1152-1178. DOI: 10.1109/TAES.2003.1261119

[5] MAHLER, P. R. S. PHD filters of higher order in target number. IEEE Transactions on Aerospace and Electronic Systems, 2007, vol. 43, no. 4, p. 1523-1543. DOI: 10.1109/TAES.2007.4441756

[6] VO, B. T., VO, B. N., CANTONI, A. The cardinality balanced multi-target multi-Bernoulli filter and its implementations. IEEE Transactions on Signal Processing, 2009, vol. 57, no. 2, p. 409 to 423. DOI: $10.1109 /$ TSP.2008.2007924

[7] VO, B. N., VO, B. T., PHUNG, D. Labeled random finite sets and the Bayes multi-target tracking filter. IEEE Transactions on Signal Processing, 2014, vol. 62, no. 24, p. 6554-6567. DOI: 10.1109/TSP.2014.2364014

[8] VO, B. T., VO, B. N. Labeled random finite sets and multi-object 
conjugate priors. IEEE Transactions on Signal Processing, 2013, vol. 61 , no. 13 , p. 3460-3475. DOI: 10.1109/TSP.2013.2259822

[9] REUTER, S., VO, B. T., VO, B. N., et al. The labeled multiBernoulli filter. IEEE Transactions on Signal Processing, 2014, vol. 62 , no. 12, p. 3246-3260. DOI: 10.1109/TSP.2014.2323064

[10] VO, B. N., MA, W. K. The Gaussian mixture probability hypothesis density filter. IEEE Transactions on Signal Processing, 2006, vol. 54, no. 11, p. 4091-4104. DOI: 10.1109/TSP.2006.881190

[11] REUTER, S., SCHEEL, A., DIETMAYER, K. The multiple model labeled multi-Bernoulli filter. In $18^{\text {th }}$ International Conference on Information Fusion. Washington, DC (USA), 2015, p. 1574-1580.

[12] GNING, A., RISTIC, B., MIHAYLOVA, L., et al. An introduction to box particle filtering. IEEE Signal Processing Magazine, 2013, vol. 30, no. 4, p. 166-171. DOI: 10.1109/MSP.2013.2254601

[13] SCHIKORA, M., GNING, A., MIHAYLOVA, L., et al. Boxparticle probability hypothesis density filtering. IEEE Transactions on Aerospace and Electronic Systems, 2014, vol. 50, no. 3, p. 1660-1672. DOI: 10.1109/TAES.2014.120238

[14] SONG, L., ZHAO, X. Box-particle cardinality balanced multitarget multi-Bernoulli filter. Radioengineering, 2014, vol. 23, no. 2 , p. 609-617.

[15] GRANSTRÖM, K., LUNDQUIST, C., ORGUNER, U. A Gaussian mixture PHD filter for extended target tracking. In $13^{\text {th }}$ Conference on Information Fusion. Edinburgh (UK), 2010, p. 1-8. DOI: $10.1109 /$ ICIF.2010.5711885

[16] GNING, A., RISTIC, B., MIHAYLOVA, L. Bernoulli particle/box-particle filters for detection and tracking in the presence of triple measurement uncertainty. IEEE Transactions on Signal Processing, 2012, vol. 60, no. 5, p. 2138-2151. DOI: 10.1109/TSP.2012.2184538

[17] LONG, Y. L., XU, H., AN, W., et al. Track-before-detect for infrared maneuvering dim multi-target via MM-PHD. Chinese
Journal of Aeronautics, 2012, vol. 25, no. 2, p. 252-261. DOI: $10.1016 / \mathrm{S} 1000-9361(11) 60385-3$

[18] LI, X. R., JILKOV, V. P. Survey of maneuvering target trackingpart V: multiple-model methods. IEEE Transactions on Aerospace and Electronic Systems, 2005, vol. 41, no. 4, p. 1255-1321. DOI: 10.1109/TAES.2005.1561886

[19] SCHUHMACHER, D., VO, B. T., VO, B. N. A consistent metric for performance evaluation of multi-object filters. IEEE Transactions on Signal Processing, 2008, vol. 56, no. 8, p. 3447-3457. DOI: 10.1109/TSP.2008.920469

\section{About the Authors ...}

Miao LI was born in 1988. He received his M.Sc. in Electronic Science and Technology from National University of Defense Technology in 2012. He is currently working toward the Ph.D. degree and interested in signal processing, small target detection and tracing.

Zaiping LIN was born in 1982. He received his Ph.D. degrees in Communication and Information System from National University of Defense Technology in 2012. His research interests include signal processing and information fusion.

Wei AN is born in1969. She is currently a Professor in National University of Defense Technology. Her research is focused on space information acquisition and processing.

Yiyu ZHOU was born in 1948. He is currently a Professor in National University of Defense Technology. His research is focused on radar signal processing, passive location technology. 